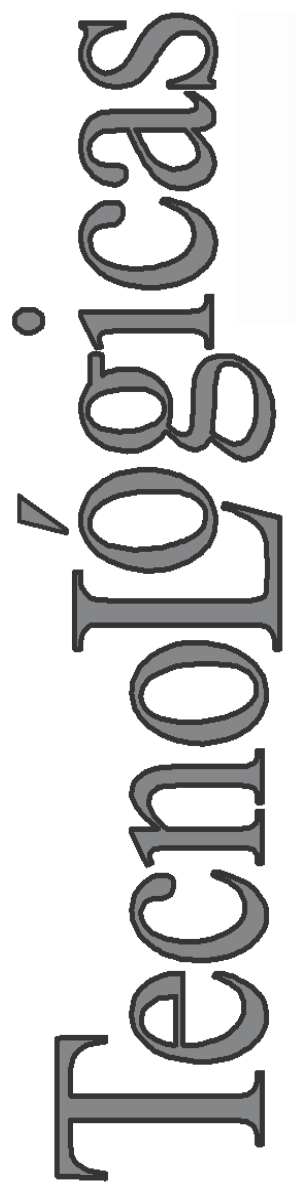

\title{
Desarrollo de un Sistema Inalámbrico para la Supervisión y Control de un Aerogenerador
}

\section{Development of a Wireless System for Monitoring and Control of a Wind Turbine}

\author{
Cristhian M. Durán-Acevedo ${ }^{1}$
} Héctor L. García-Sierra ${ }^{2}$

1 Facultad de Ingeniería y Arquitectura, Grupo de investigación en Sistemas Multisensoriales y Reconocimiento de Patrones, Universidad de Pamplona, Pamplona-Colombia cmduran@unipamplona.edu.co

2 Facultad de Ingeniería y Arquitectura, Grupo de investigación en Sistemas Multisensoriales y Reconocimiento de Patrones, Universidad de Pamplona, Pamplona-Colombia hgarcias77@hotmail.com 


\section{Resumen}

El artículo presenta el uso de una tecnología de comunicación inalámbrica a través del protocolo ZigBee, mediante la implementación de los módulos XBee S2B. La comunicación inalámbrica fue implementada sobre un prototipo de sistema de aerogenerador eléctrico (es decir, para generación de energía eólica), con el objetivo de controlar en forma automática diferentes variables como son: La dirección del viento, temperatura ambiente, humedad y velocidad del motor. Los XBee fueron acondicionados por medio de una tarjeta Arduino Mega ADK, en la cual se adquirieron las señales generadas por diferentes sensores y posteriormente fueron enviadas inalámbricamente. La programación del módulo Arduino y el monitoreo de cada una de las variables fueron realizadas a través del software Labview. El estudio también fue desarrollado con el fin de explorar nuevas tecnologías de comunicación inalámbrica, el cual es de gran utilidad en sistemas interoperables para monitorear, controlar y automatizar diferentes procesos. Como resultado, las pruebas de funcionamiento con el sistema inalámbrico fue estable y la transmisión de datos confiable.

\section{Palabras clave}

Aerogenerador; Arduino; comunicación inalámbrica; Labview; XBee.

\section{Abstract}

This article presents the use of a wireless communication technology through the ZigBee protocol, by implementing XBee S2B. Wireless communication was implemented on a wind turbine prototype (i.e. wind power generation) in order to controlling variables automatically, such as: Direction of the wind, temperature, humidity and velocity engine. The XBee were conditioned using a Mega ADK Arduino card, which the signals generated were acquired by several sensors and subsequently sent wirelessly. The programming and monitoring of Arduino module with each of the variables was performed through Labview software. The study was also conducted in order to explore new technologies for wireless communication, which is useful in interoperable systems to monitor, control and automate different processes. As a result, the performance test with the wireless system was stable and data transmission was reliable.

\section{Keywords}

Wind turbine; Arduino; wireless communication; Labview; XBee. 


\section{INTRODUCCIÓN}

Las tecnologías inalámbricas han adoptado en el transcurso del tiempo una manera más sencilla y cómoda de utilizar toda clase de dispositivos, con el fin de mejorar las comunicaciones en general. Este trabajo aborda la tecnología inalámbrica ZigBee, basada en el estándar IEEE 802.15.4 de comunicaciones inalámbricas. ZigBee es una tecnología adoptada por ZigBee Alliance, el cual es desarrollado para cubrir las necesidades de seguridad, fiabilidad, flexibilidad, bajo coste y poco consumo eléctrico en las diferentes áreas del control de procesos. Es similar al modelo de referencia OSI (Open System Interconnection), donde es formada por varias capas independientes entre sí y con funciones específicas (Dubendorfer \& Ramsey, 2012). También permite acoplar funciones inteligentes que mejoran la eficiencia de diferentes procesos y es posible comunicar varios dispositivos haciendo que trabajen más eficientemente entre sí.

Los módulos ZigBee están compuestos de un transmisor y un receptor que usan muy poca energía para funcionar y tienen como objetivo las aplicaciones que requieren comunicaciones seguras con una mínima tasa de datos. Con casi ya una década y con millones de implementaciones por año, la norma ZigBee cuenta con la más amplia variedad de aplicaciones. Algunos trabajos reportan diferentes estudios a partir de esta tecnología, donde algunos de ellos han sido implementados para el sector de la salud, como el monitoreo del corazón (Zulkifli et al., 2012; Kioumars et al., 2011), en la adquisición en tiempo real de fuerzas de reacción para personas con miembros inferiores amputados (Stalin, et al., 2013); también en aplicaciones industriales, tales como: Control ambiental (Rodríguez et al., 2011), para inspecciones con sistemas robóticos (Yusoff et al., 2012), control de abastecimiento de agua potable (Durán \& Iturriago, 2011) entre otros.

Algunos estudios van conducidos a utilizar Zigbee para el diseño de aplicaciones mediante "Smart Energy", en el entorno comercial (Dae et al., 2010), y para el control automático del consumo de energía en el hogar, mediante el monitoreo de electrodomésticos (Mrazovac et al., 2012). Es por esta razón que la tecnología ZigBee Smart Energy actualmente se está utilizando ampliamente, debido 
a que ayuda sobre todo a crear viviendas más ecológicas dando a los consumidores una completa información y la automatización necesaria, para reducir fácilmente el consumo y así ahorrar gran cantidad de dinero.

En la actualidad no se presentan reportes sobre aplicaciones de Zigbee Smart Energy en cuanto a la aplicación desarrollada en este estudio. Por tal motivo se podría formular un diseño de bajo costo utilizando módulos XBee y XBee-PRO en una aplicación de aerogeneradores de energía, con el fin de desarrollar cualquier dispositivo de uso inteligente de energía que incluya las siguientes características: Un portal de servicios energéticos, dispositivos de medición, termostatos programables, controladores de carga y amplificadores. Para esta aplicación se requiere y es necesario asegurar que los dispositivos de energía inteligentes sean capaces de realizar comunicaciones sencillas y seguras entre los diferentes elementos que hacen parte del proceso.

Es importante aclarar que los módulos actuales XBee MaxStrem son una familia de Digi International, el cual es un producto certificado por la Zigbee Alliance. Los módulos XBee soportan el protocolo 802.15.4, mientras que los módulos XBee PRO soportan el protocolo ZigBee. Los módulos de la serie 2 en este caso pueden funcionar como Zigbee, con el perfil de Smart Energy o como Digimesh. Comparando con otras tecnologías inalámbricas, actualmente se está empleando al igual que el XBee tecnologías con módulos Bluetooth, los cuales son utilizados para el control y automatización de procesos industriales (Durán \& Miranda, 2012).

El artículo va dirigido a implementar un módulo de comunicación inalámbrica a un sistema aerogenerador de energía, en el cual se analizan una serie de variables que pueden ser observadas desde una interfaz de usuario gráfica. El software utilizado para la instrumentación virtual fue el Labview y el sistema de control fue realizado por medio de una tarjeta Arduino Mega ADK, en la cual se reciben las señales generadas por los diferentes sensores que se utilizaron en el presente estudio. 


\subsection{Diferencias entre XBee y otras Tecnologías}

Existen muchas alternativas de comunicaciones inalámbricas asequibles dependiendo de los diseñadores; comparando XBee con Bluetooth y Wi-Fi de algunos de los estándares más populares que comparten la banda de $2.4 \mathrm{GHz}$. Los parámetros incluyen el estándar que debe seguirse a nivel de la capa dos (es decir, el formato de la trama), la máxima velocidad de transmisión, el consumo de corriente típica en transmisión y en "standby", los requisitos de memoria de programa para un dispositivo típico, aplicaciones y opciones de conexión de la red entre otras características (Shafi et al., 2002).

\subsection{Topología de Red para Clúster}

Para seleccionar el tipo de topología de red se debe considerar la ubicación de los puntos de acceso o Gateways y la distancia mínima desde la infraestructura de la red al nodo o dispositivo final. Una opción de topología es una red simple en estrella donde un punto de acceso central tiene conectados varios dispositivos finales; ésta es una configuración ideal para Wi-Fi siempre y cuando las distancias desde los puntos de acceso a los dispositivos sean menores de 100 metros. Si es necesaria una distancia adicional, una topología de árbol podría ser utilizada, por lo cual es posible usar repetidores Wi-Fi o ruteadores XBee Si la fiabilidad de la red es importante, entonces una red de malla le ofrece trayectorias redundantes desde un nodo final a través de múltiples ruteadores hasta el Gateway. Esto brinda fiabilidad de red en caso de que un ruteador falle.

Ya que los módulos XBee permiten ser utilizados en diferentes topologías de red, tales como: Punto a punto, red en estrella, árbol de clúster y malla (mesh), cada topología admite la comunicación entre diferentes dispositivos que se encuentran fuera de su rango de transmisión, teniendo en cuenta que para la mayoría de las topologías deben estar conectados por nodos intermedios.

Para el caso del control aerogenerador en aplicaciones de energía eólica, es necesario conocer el medio donde se ubicaría el sistema propuesto. Por tal motivo a parte del sitio es necesario de- 
terminar la distancia y la topología. Es por esta razón que, para que el sistema sea altamente confiable se recomienda seguir las especificaciones de los diferentes módulos inalámbricos: Por ejemplo, para distancias mayores de 100 metros la tecnología XBee da la opción de incrementar la distancia a un valor de 1000 metros en línea de vista (topología punto a multipunto).

En este estudio nosotros proponemos que para el control de las turbinas eólicas, es útil ubicar un sistema XBee de la serie 2 para formar redes de sensores inalámbricos. Las redes de sensores recogen eficientemente la información relacionada sobre el estado de la operación del sistema eólico, como por ejemplo: Los elementos de la parte mecánica y la unidad eléctrica, así mismo información sobre el sitio de operación. En una red de sensores cada aerogenerador puede representar una red inalámbrica, la cual podría enviar la información a un centro local remoto a través de enrutadores inalámbricos. Dentro de las topologías más fiables y asequibles para los parques eólicos en alta mar como en tierra, con una mayor seguridad y eficiencia se pueden destacar: Estrella, árbol de clúster y malla.

Mediante los módulos XBee PRO de la Serie 2 y la placa base de Arduino, se llegaría a obtener una gran eficiencia y fiabilidad mediante la topología tipo malla, debido a que no existiría ninguna perturbación en las comunicaciones, en caso de que falle algún dispositivo existe el otro que se haría cargo del tráfico y el mantenimiento sería muy bajo. Solo la única desventaja se centraría en el costo de los materiales, ya que se aumentarían ampliamente los dispositivos inalámbricos, por lo cual sería conveniente determinar la ubicación del control principal.

\section{MATERIALES Y MÉTODOS}

Los módulos inalámbricos utilizados para realizar la comunicación bidireccional para la trasmisión y recepción de los datos adquiridos a través del PC (Computador personal) y el Arduino Mega ADK (es decir, base del aerogenerador), fue mediante dispositivos XBee PRO S2B con capacidad de acondicionamiento de hasta 8 entradas y salidas digitales; el cual proporciona la ventaja 
de tener gran flexibilidad en la configuración del sistema (Mahanti et al., 2007).

Los dispositivos XBee utilizados en este estudio se comunican con un microcontrolador mediante comunicación UART (Universal Asynchronous Receiver-Transmitter). Este dispositivo permite eliminar el cable de comunicación entre el PC y dispositivos a controlar, utilizando la placa Arduino MEGA ADK (Batista et al., 2013).

\subsection{Arduino MEGA ADK}

\subsubsection{Módulos inalámbricos XBee PRO de la serie S2b}

Inicialmente se configuraron los módulos XBee mediante el programa X-CTU que hace parte del módulo inalámbrico y fue necesario configurarlo en el modo transparente con los siguientes parámetros: Se configuró como router y como coordinador, de esta forma se asegura una comunicación punto a punto entre los dos dispositivos con una velocidad de 9600 Baudios, el cual viene configurado el Arduino por defecto. La tarjeta utiliza un microcontrolador, donde la integra un host USB y un Arduino Mega 2560.

\subsubsection{Configuración Arduino y XBee PRO de la serie S2b}

La Fig. 1 ilustra la configuración para adaptar la tarjeta con los módulos XBee. Cada módulo XBee viene configurado con un ID (Identificador de la red personal: Número 8888), y a su vez configurados a 9600 baudios, 8 bits de datos, sin paridad y 1 bit de paro. El XBee Shield tiene un par de puentes para definir si la comunicación serial se realiza hacia el puerto USB o hacia el módulo XBee. Como primera prueba se configuró para interactuar con el puerto USB, el cual ubica ambos puentes en la posición externa de los pines (Ganssle et al., 2012).

\subsubsection{Diseño del aerogenerador}

Para realizar las pruebas con los módulos inalámbricos se desarrolló un prototipo de aerogenerador, el cual fue basado en el diseño de los tipos de aerogeneradores de eje horizontal de tres palas. 


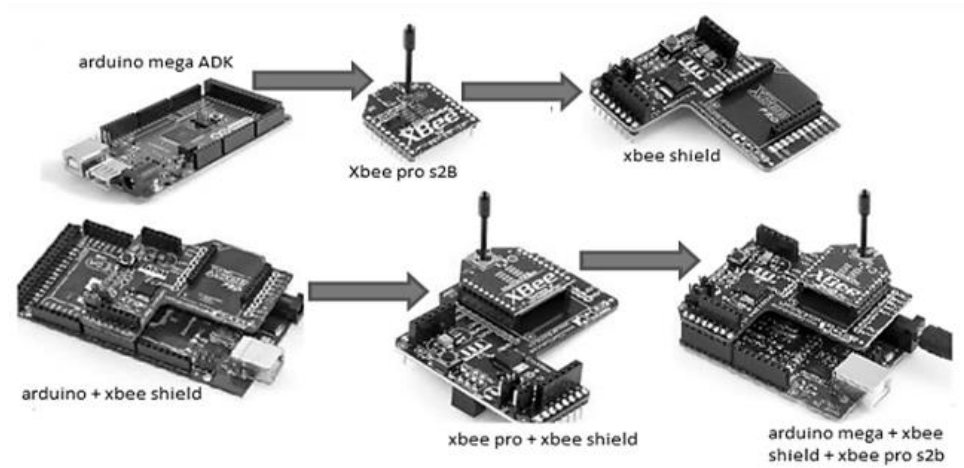

Fig. 1. Configuración de los módulos XBee con la tarjeta Arduino MEGA ADK. Fuente: Autores

A continuación se relacionan los materiales utilizados para el desarrollo del prototipo de aerogenerador:

1. Un Tubo de PVC (Polyvinyl chloride) de 3 pulgadas

2. División (T) de PVC de alta presión

3. Reductor PVC de 3 a 2 pulgadas

4. Motor DC Mitsubishi (24 Voltios)

5. 8 Sensores finales de carrera (Para determinar la posición del aerogenerador)

6. Sensor de temperatura (Para el monitoreo de la temperatura ambiente)

7. Sensor de humedad HSM20G (Para el monitoreo de la humedad ambiente)

8. Sensor CNY 70 (Para determinar los RPM del Motor)

9. Bricolaje en general

\subsubsection{Diagramas de flujo para configurar el Arduino}

En la Fig. 2 se ilustra el diagrama de flujo para configurar el Arduino desde Labview. En el controlador se configura la velocidad de transmisión (es decir, 9600 baudios), la cual debe ser la misma a la configurada en Labview. Luego el puerto es acondicionado para realizar la comunicación y posteriormente se le asignan las librerías de Labview al microcontrolador de Arduino (ver Fig. 2 ). 


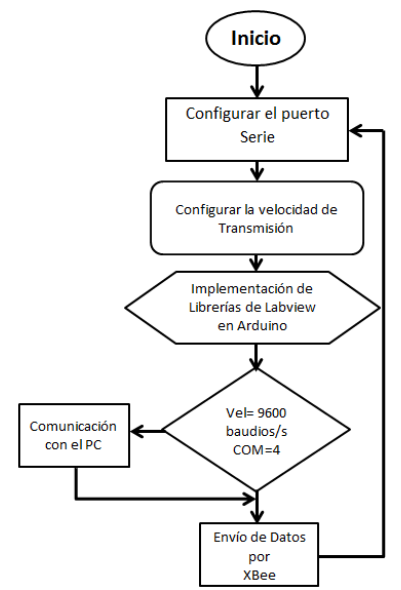

Fig. 2. Diagrama para configurar el Arduino desde Labview. Fuente: Autores

Tal y como se observa en el diagrama de la Fig. 3 el programa inicia con la configuración de los módulos XBee con el objetivo de realizar una comunicación bidireccional (punto a punto), y así enviar y recibir los datos leídos por los sensores que se encuentran en la base del aerogenerador, dependiendo de la variable leída. Los sensores en esta etapa se activan y envían una señal por medio del Arduino. Los sensores finales de carrera llamados FDC indican en qué dirección se encuentra el aerogenerador. Para calcular las RPM del motor se utilizó un sensor CNY 70, donde cada vez que se activaba se genera un registro en el programa y se incrementaba. Si el valor de las RPM era mayor que 200 se activa el sistema de control de velocidad. Para el caso del monitoreo de la temperatura y la humedad el cual se realizó por medio del sensor HSM20G, los valores fueron presentados en el PC por medio de la interfaz de usuario diseñada en Labview.

\subsubsection{Acondicionamiento de los sensores al aerogenerador}

Para monitorear la dirección o posición en la que se encuentra el aerogenerador con respecto al viento, se utilizaron 8 sensores finales de carrera ubicados en un disco, con el objetivo de obtener mayor resolución o exactitud en la dirección en la que se ubica el aerogenerador (ver Fig. 4). 
[404] Durán \& García / Desarrollo de un Sistema Inalámbrico para la Supervisión y Control de un Aerogenerador

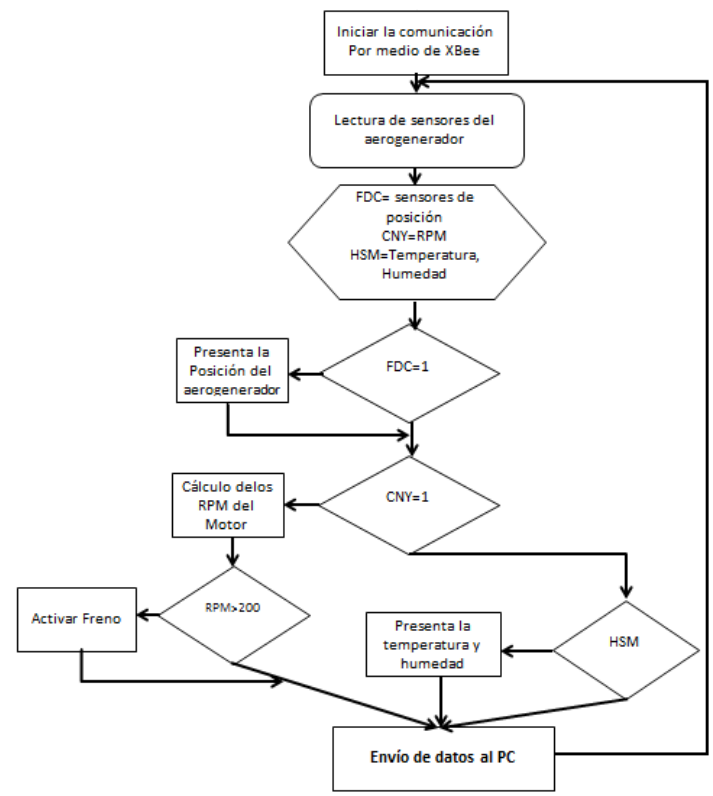

Fig. 3. Diagrama de configuración de los sensores en Labview. Fuente: Autores

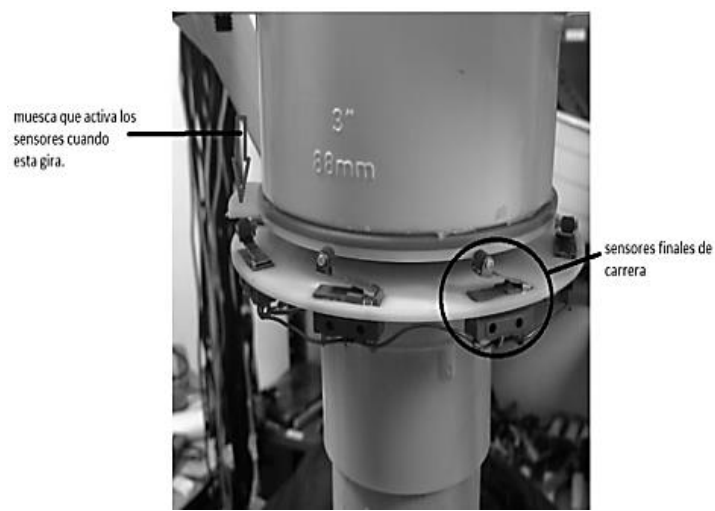

Fig. 4. Acondicionamiento de los finales de carrera al aerogenerador.

Fuente: Autores

Para medir la velocidad del motor se utilizó un sensor CNY70 para enviar los pulsos en el momento dado que el motor de una vuelta. Se diseñó un encoder para enviar un pulso cada vez que se 
dé un giro. En algunos casos la velocidad del motor era demasiado rápida y el sensor no alcanzaba a adquirir los datos correctamente, debido a que el voltaje no variaba y se mantenía siempre en el valor de los 3.3 voltios. Lo anterior hacía que la tarjeta Arduino no reconociera los cambios de flanco para realizar dicho conteo y así efectuar el cálculo de la velocidad del motor. Para solucionar dicho inconveniente se utilizó una compuerta de disparo para garantizar que siempre que existiera un cambio de flanco, se envíe un " 1 " o un "0" para generar el conteo.

\section{RESULTADOS}

Para asegurar el correcto funcionamiento de los módulos XBee, se utilizó nuevamente el programa X-CTU, donde se verificó la configuración de los dispositivos y el puerto del computador, estableciendo de esta forma la comunicación entre los módulos. En la Fig. 5 se ilustra una interface de usuario gráfica realizada en Labview, en la cual fue posible monitorear cada una de las señales enviadas por el aerogenerador, donde se realizan los cálculos con la información proveniente de cada uno de los sensores y de esta forma se garantizaba el buen funcionamiento del sistema.

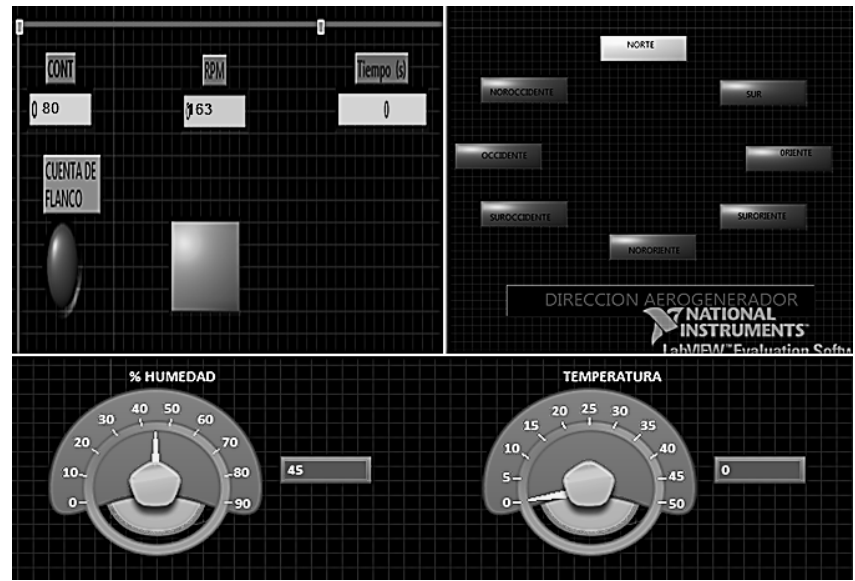

Fig. 5. Implementación y funcionamiento del control manual con la interfaz de usuario. Fuente: Autores 
Tal y como se ilustra en la Fig. 6, en cada una de las gráficas (con auto-escala) fue posible observar el comportamiento de la temperatura y la humedad medida en el aerogenerador, a su vez supervisar el comportamiento del contador encargado de detectar el número de vueltas del motor del aerogenerador.
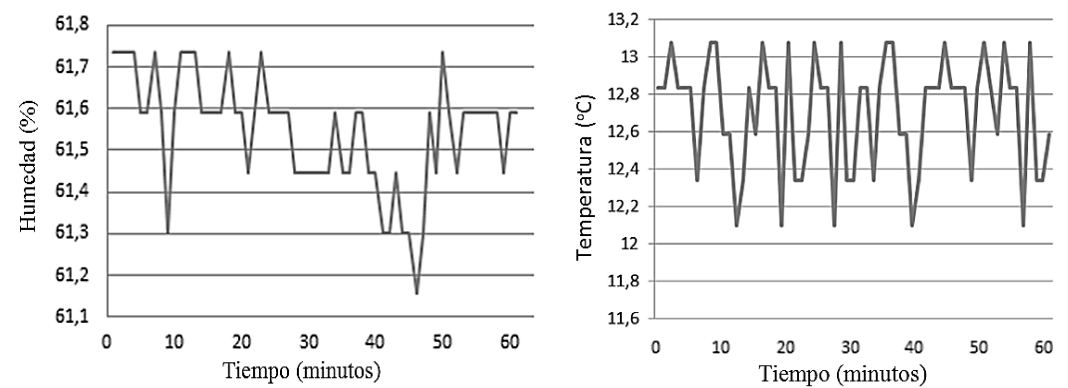

Fig. 6. Gráficas de pruebas de campo (Temperatura y Humedad). Fuente: Autores

A través de la Fig. 7 se observa el aerogenerador funcionando en diferentes pruebas de campo realizadas.

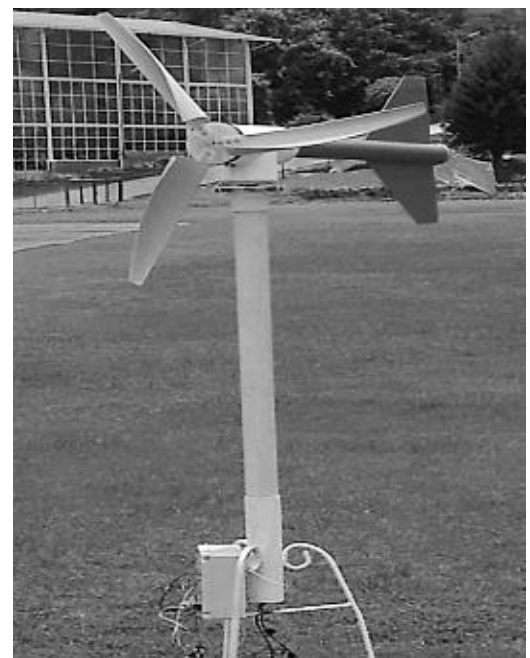

Fig. 7. Implementación del aerogenerador, pruebas de campo. Fuente: Autores 
El control del frenado del sistema se realizó mediante un potenciómetro virtual diseñado en Labview, el cual controla una salida PWM de la tarjeta Arduino. El método de frenado aunque no fue muy robusto, se realizó por medio de un transistor IRF 540, el cual estaba conectado al motor del aerogenerador suministrando un voltaje en inversa en caso de ser accionado por medio del accionamiento. La utilización del módulo de supervisión y control permitió desarrollar una interfaz gráfica flexible al usuario, ya que esta herramienta permite personalizar cada uno de los instrumentos, logrando con ello tener una mejor apariencia y vista para la supervisión del proceso.

La adquisición de las muestras del aerogenerador se realizó a través de una carga con bombilla de 12 VDC, llegando a generar una potencia de 30 vatios (W) aproximadamente a una velocidad de $160 \mathrm{rpm}$. El alcance obtenido en las pruebas de campo abierto fue de 70 metros y desde el control desde un sitio cerrado llegó a 30 metros aproximadamente. En la Fig. 8. Se observa el diagrama en bloques del proceso del aerogenerador donde fue implementada la comunicación inalámbrica.

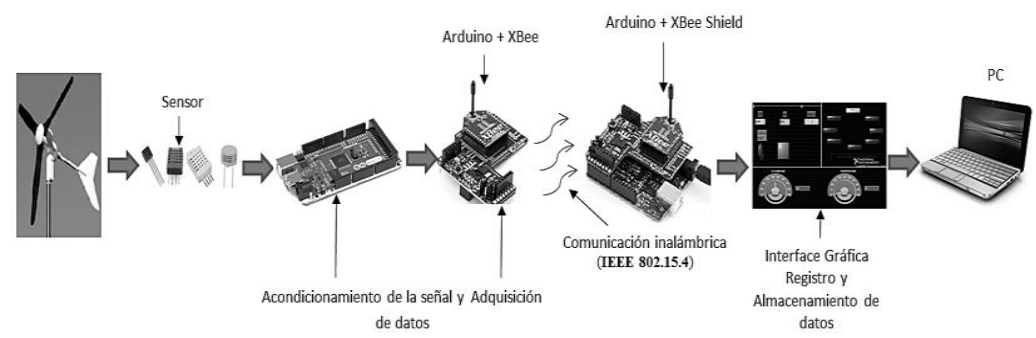

Fig. 8. Diagrama en bloques del sistema

\section{CONCLUSIONES}

Con el desarrollo de este estudio se ha introducido el estándar basado en tecnología ZigBee como una herramienta versátil para la automatización de procesos a distancia, aplicado a sistemas generadores de energía eléctrica. Se determinó que los módulos XBee son muy sensibles a fluctuaciones en la fuente de alimentación, ya que los picos de voltaje de la fuente afectaron a los disposi- 
tivos en el momento del funcionamiento, por lo que fue muy importante optimizar el filtrado en la alimentación de cada uno de estos dispositivos.

El uso de la tarjeta Arduino MEGA facilitó la conexión con el entorno de Labview, a través del uso de varias herramientas de instrumentación virtual que pueden ser acopladas a diferentes módulos inalámbricos. El prototipo del aerogenerador diseñado en este estudio se puede utilizar para analizar e identificar las variables que componen a este sistema y así poderlo relacionar con un aerogenerador real, teniendo en cuenta el tipo de topología de red a utilizar. Aunque la aplicación del aerogenerador permitió validar el correcto funcionamiento de la comunicación inalámbrica, para un desarrollo completo del aerogenerador se requiere calcular la velocidad del viento, el uso de baterías, un inversor DC/AC, entre otras variables.

\section{AGRADECIMIENTOS}

Los integrantes del artículo agradecen a la Universidad de Pamplona y la Convocatoria 50 años, por los recursos dados para la realización del proyecto del aerogenerador con tecnología XBee.

\section{REFERENCIAS}

Batista, N.C., Melício, R., Matias, J.C.O., Catalão, J.P.S. (2013). Photovoltaic and wind energy systems monitoring and building/home energy management using ZigBee devices within a smart grid, Energy, 49, 306-315.

Dae, M.H., Jae, H.L. (2010). Smart home energy management system using IEEE 802.15.4 and zigbee, IEEE Transactions on Consumer Electronics, 56(3), 1403-1410.

Dubendorfer, C.K., Ramsey, B.W., Temple, M.A. (2102). An RF-DNA verification process for ZigBee networks, Military Communications Conference, MILCOM 2012.

Durán, A.C.M., Miranda, R.A. (2012). Comunicación inalámbrica basada en tecnología Bluetooth para la automatización de procesos industriales, Revista el Hombre y la Máquina, 39, 26-32. 
Durán, A.C.M., Iturriago, A.X. (2012). Automatización de un Sistema de Suministro de Agua Potable, A través de la tecnología ZigBee, Revista colombiana de tecnologías de avanzada, 2, 36-42.

Ganssle, J. (2012). Foreword to the Hands-on XBee Lab Manual, the Hands-on XBEE Lab Manual, 11-12.

Kioumars, A.H., Liqiong, Tang. (2011). Wireless network for health monitoring: heart rate and temperature sensor, Fifth International Conference on Sensing Technology (ICST), 362-369.

Mahanti, A., Williamson, C., Arlitt, M., Mahantit, A. (2007). Comparing Wired-side and Wireless-side WLAN Monitoring Techniques: A Case Study32nd IEEE Conference on Local Computer Networks, 901-910.

Mrazovac, B., Bjelica, M.Z., Kukolj, D., Todorovic, B.M., Teslic, N. (2012). A human detection method $\mathrm{f}$ or residential smart energy systems based on Zigbee RSSI changes, IEEE International Conference on Consumer Electronics (ICCE), 110-111.

Rodriguez, M.G., Ortiz Uriarte, L.E., Yi, J., Yoshii, K., Beckman, P.H. (2011). Wireless sensor network for data-center environmental monitoring, Fifth International Conference on Sensing Technology (ICST), 533-537.

Shafi, M., Ogose, S., Hattori, T. (2002). Visions of Wireless Communications Applications in the 21st Century, Wireless Communications in the 21st Century, pp. 22.

Stalin, M.B., Christopher, L. (2013). Development of an Insole System for Real-time Capture of Ground Reaction Forces in Lower-limb Amputees, Biomedical Engineering Conference (SBEC), 137-138.

Yusoff, M.F., Ibrahim, B.S.K.K., Hamzah, H., Kadir, H.A. (2012). Development of Air Conditional Route Wireless Inspection Robot Original Research Article, Procedia Engineering, 41, 874-880.

Zulkifli, N.S.A., Harun, F.K.C., Azahar, N.S. (2012). XBee wireless sensor networks for Heart Rate Monitoring in sport training, 2012 International Conference on Biomedical Engineering (ICoBE), 441-444. 